\title{
Nano-skeletal formation from decagonal quasicrystalline surface
}

Shashank Shekhar Mishra ${ }^{1}$, Thakur Yadav ${ }^{1}$, Nilaykrishna Mukhopadhyay ${ }^{1}$, Onkarnath Srivastava ${ }^{1}$

${ }^{1}$ Department Of Physics, Banaras Hindu University, Varanasi, Varanasi, India E-mail: smishra747@gmail.com

Quasicrystal (QC) is a new form of material that differ from amorphous and crystalline materials, as it possess a perfect long range translational and orientational ordering though it lacks of periodicity in atomic structure [1]. In addition to stability at high temperature and high strength and also the participation of catalytic elements (such as $\mathrm{Fe}, \mathrm{Cu}, \mathrm{Co}, \mathrm{Cr}, \mathrm{Ni}$, etc. in many Al-based QCs), provides the additional features to QCs to be used as a catalyst [2]. The effectiveness of catalysis depends critically on the high interface area between the catalyst and the reactant (gases or liquids) [3]. Consequently, research has recently been undertaken on the processing of catalysts with nanometer sized porous structure. Here, we have studied the surface microstructure, chemical composition and structural characteristics of the conventionally solidified Al-Cu-Co alloys with nominal composition of Al65Cu15Co20 and Al65Cu20Co15 during the chemical leaching. The surface of polygrain Al-CuCo decagonal quasicrystals was leached with 2.5 mole $\mathrm{Na} 2 \mathrm{CO} 3$ solution for various leaching times. The resulting surfaces were investigated by $\mathrm{x}$-ray diffraction, scanning electron microscopy and transmission electron microscopy techniques. The energy dispersive $\mathrm{x}$-ray analysis was employed to find the chemical composition of the leached surface. The results show that the leaching treatment changes the crystallographic structure of the leached surface from the quasicrystalline to crystalline. The formation of the skeletal structure was observed at the surface due to removal of Al from the lattice sites. This skeletal structure was consisting of finely distributed nano-particles of metal and their associated oxides structure (Cu, $\mathrm{Co}, \mathrm{Cu} 2 \mathrm{O}$ and $\mathrm{CuO}$ ) with pores. The polycrystalline nature of these of these nano particles was confirmed by the selected area diffraction patterns. Attempts will be made to discuss the mechanism of the formation of nanoparticles.

[1] Shechtman, D. \& Blech, I.A. (1985). Metallurgical Transactions A. 16, 1005-1012.

[2] Tsai, A. P. \& Yoshimura, M. (2001). Appl. Catal. A. 214, 237-341.

[3] Kameoka, S. et al. (2014). Sci. Technol. Adv. Mater. 15, 014801 1- 7.

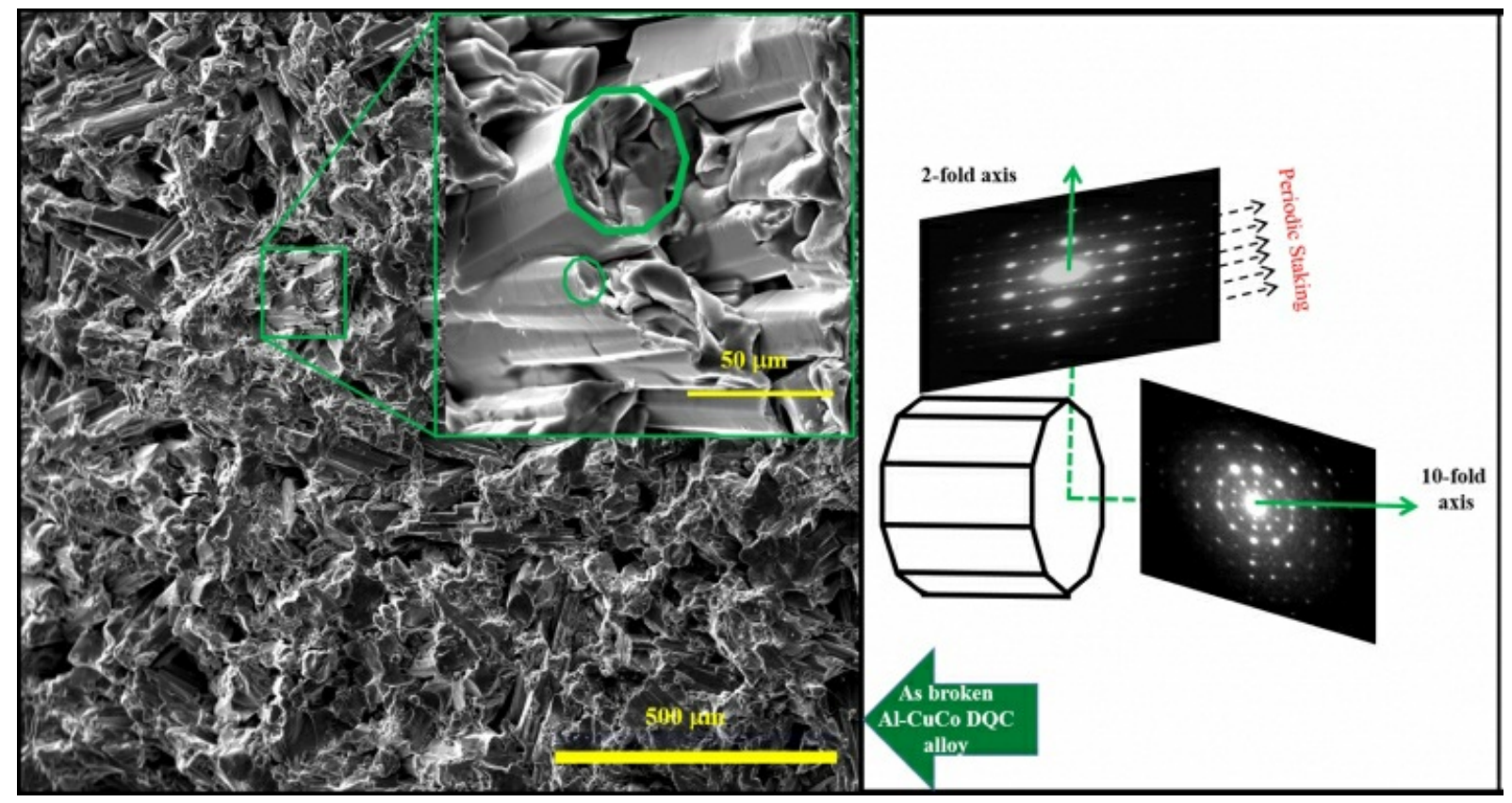

Keywords: Skeletal structure, Quasicrystal, Nano-particles 